\title{
Eigenvalues of a Class of Singular Boundary Value Problems of Impulsive Differential Equations in Banach Spaces
}

\author{
Daliang Zhao and Yansheng Liu \\ School of Mathematical Sciences, Shandong Normal University, Jinan, Shandong 250014, China \\ Correspondence should be addressed to Yansheng Liu; yanshliu@gmail.com
}

Received 26 May 2013; Accepted 28 October 2013; Published 23 February 2014

Academic Editor: Gestur Ólafsson

Copyright (C) 2014 D. Zhao and Y. Liu. This is an open access article distributed under the Creative Commons Attribution License, which permits unrestricted use, distribution, and reproduction in any medium, provided the original work is properly cited.

\begin{abstract}
This paper is devoted to investigating the eigenvalue problems of a class of nonlinear impulsive singular boundary value problem in Banach spaces: $\mu x^{\prime \prime}+f(t, x)=0, t \in(0,1), t \neq t_{i} ;\left.\Delta x\right|_{t=t_{i}}=\alpha_{i} x\left(t_{i}-0\right), i=1,2, \ldots, k ; a x(0)-b x^{\prime}(0)=\theta ; c x(1)+d x^{\prime}(1)=\theta$, where $\theta$ denotes the zero element of Banach space, $\left.\Delta x\right|_{t=t_{i}}=x\left(t_{i}+0\right)-x\left(t_{i}-0\right), \alpha_{i}>-1, a, b, c, d \in R^{+}, \gamma=a c+a d+b c>0, \mu$ is a parameter, and $f(t, x)$ may be singular at $t=0,1$ and $x=\theta$. The arguments are mainly based upon the theory of fixed point index, measure of noncompactness, and the special cone, which is constructed to overcome the singularity.
\end{abstract}

\section{Introduction}

Consider the following eigenvalue problems of singular boundary value problem (SBVP) with impulse in Banach space $E$ :

$$
\begin{aligned}
\mu x^{\prime \prime}+ & f(t, x)=0, \quad t \in(0,1), t \neq t_{i} ; \\
\left.\Delta x\right|_{t=t_{i}}= & \alpha_{i} x\left(t_{i}-0\right), \quad i=1,2, \ldots, k ; \\
& a x(0)-b x^{\prime}(0)=\theta ; \\
& c x(1)+d x^{\prime}(1)=\theta,
\end{aligned}
$$

where $\theta$ denotes the zero element of Banach space $E,\left.\Delta x\right|_{t=t_{i}}=$ $x\left(t_{i}+0\right)-x\left(t_{i}-0\right), 0=t_{0}<t_{1}<t_{2}<\cdots<t_{k}<t_{k+1}=1$, $\alpha_{i}>-1, a, b, c, d \in R^{+}, \gamma=a c+a d+b c>0, \mu$ is a parameter, $f(t, x)$ may be singular at $t=0,1$ and $x=\theta$.

During the last few decades, the existence of positive solution of nonlinear singular boundary value problems has gained considerable popularity (see [1-12] and references therein). In recent years, there were also a lot of papers which dealt with eigenvalue problems (see [13-27]). Some of them considered singular case (see, for instance, [13-16, 18, 19], etc.).
On the other hand, as we know, the theory of impulsive differential equations has found its extensive applications in realistic mathematical modeling of a wide variety of practical situations such as physics, chemical technology, population dynamics, biotechnology, and economics. It has emerged as an important area of investigation in recent years (see [2836] and references therein).

To the best of our knowledge, there is no paper studying the eigenvalue problems of the impulsive singular boundary value problem in Banach spaces. The main purpose of this paper is to fill this gap. By using the theory of fixed point index, measure of noncompactness, and the special cone which is constructed to overcome the singularity, we investigate the existence of eigenvalues of (1).

The main features of the present paper are as follows. By virtue of a special transformation, we first convert (1) into another solvable form such that the associated operator can be used to overcome the influence of impulse and parameter $\mu$. Then a special cone is constructed to deal with the singularity of (1).

This paper is organized as follows. In Section 2, we provide some basic definitions, preliminaries facts, and lemmas. Meanwhile, some transformations are introduced to convert (1) into another solvable form. In Section 3, the main results 
are presented and proved. Finally, an example is worked out to demonstrate the application of the main result.

\section{Preliminaries and Conversion of (1)}

Let $P$ be a normal solid cone of real Banach space $E$. Without loss of generality, suppose the normal constant is 1 . Let $P^{*}$ denote the dual cone of $P, J=[0,1]$, and $P_{r}=\{x \in P:\|x\|<$ $r\}, \bar{P}_{r}=\{x \in P:\|x\| \leq r\}(r>0)$. Denoted by $C[J, E]$ the Banach space of all continuous functions $x: J \rightarrow E$ with norm $\|x\|_{c}=\max _{t \in J}\|x(t)\|$.

We define $P C[J, E]=\{x: x$ is a map from $J$ into $E$ such that $x(t)$ is continuous at $t \neq t_{i}$ and left continuous at $t=t_{i}$ and its right limit at $t=t_{i}$ (denoted by $x\left(t_{i}^{+}\right)$) exists for $i=1,2, \ldots, m\}$, and $P C^{1}[J, E]=\left\{x \in P C[J, E]: x^{\prime}(t)\right.$ is continuous at $t \neq t_{i}$ and left continuous at $t=t_{i}$ and the right limit at $t=t_{i}$ (denoted by $x^{\prime}\left(t_{i}^{+}\right)$) exists for $\left.i=1,2, \ldots, m\right\}$.

Let $Y=\left\{y \in C^{1}[J, E]: a y(0)-b y^{\prime}(0)=\theta, c y(1)+\right.$ $\left.d y^{\prime}(1)=\theta\right\}$, and $X=\left\{x \in P C^{1}[J, E]:\left.\Delta x\right|_{t=t_{i}}=\alpha_{i} x\left(t_{i}-\right.\right.$ $0),\left.\Delta x^{\prime}\right|_{t=t_{i}}=\alpha_{i} x^{\prime}\left(t_{i}-0\right), a x(0)-b x^{\prime}(0)=\theta, c x(1)+d x^{\prime}(1)=$ $\theta\}$. It is well known that $Y$ is a Banach space with the norm $\|y\|_{1}=\max \left\{\max _{t \in J}\|y(t)\|, \max _{t \in J}\left\|y^{\prime}(t)\right\|\right\}$.

Evidently, $P C[J, E]$ and $P C^{1}[J, E]$ are Banach spaces with norm $\|x\|_{P C}=\sup _{t \in J}\|x(t)\|$ and $\|x\|_{P C^{1}}=\max \left\{\sup _{t \in J}\|x(t)\|\right.$, $\left.\sup _{t \in J}\left\|x^{\prime}(t)\right\|\right\}$, respectively. Furthermore, $X$ is a closed subspace of $P C^{1}[J, E]$.

For each $y \in Y$, let $x(t)=\prod_{0<t_{i}<t}\left(1+\alpha_{i}\right) y(t)$; it is easy to see $x \in X$. Conversely, for each $x \in X$, let $y(t)=$ $x(t) / \prod_{0<t_{i}<t}\left(1+\alpha_{i}\right)$; then $y \in Y$.

We convert (1) into another solvable form first.

Lemma 1. If $x \in X$ is a solution of impulsive $S B V P$ (1), then $y(t)=x(t) / \prod_{0<t_{i}<t}\left(1+\alpha_{i}\right)$ satisfies SBVP:

$$
\begin{gathered}
\mu y^{\prime \prime}+\frac{1}{\prod_{0<t_{i}<t}\left(1+\alpha_{i}\right)} f\left(t, \prod_{0<t_{i}<t}\left(1+\alpha_{i}\right) y(t)\right)=0, \\
\quad t \in(0,1) ; \\
a y(0)-b y^{\prime}(0)=\theta ; \\
c y(1)+d y^{\prime}(1)=\theta .
\end{gathered}
$$

Conversely, if $y \in Y$ is a solution of SBVP (2), then $x(t)=$ $\prod_{0<t_{i}<t}\left(1+\alpha_{i}\right) y(t)$ is a solution of impulsive SBVP (1).

Lemma 2. $y \in Y$ is a solution of SBVP (2) if and only if $y$ is a solution of the integral equation:

$$
\begin{aligned}
\mu y(t)= & \int_{J} \frac{1}{\prod_{0<t_{i}<s}\left(1+\alpha_{i}\right)} G(t, s) \\
& \times f\left(t, \prod_{0<t_{i}<s}\left(1+\alpha_{i}\right) y(s)\right) d s,
\end{aligned}
$$

where

$$
G(t, s)= \begin{cases}\gamma^{-1}(a t+b)(c(1-s)+d), & 0 \leq t \leq s \leq 1, \\ \gamma^{-1}(a s+b)(c(1-t)+d), & 0 \leq s \leq t \leq 1 .\end{cases}
$$

By Lemmas 1 and 2, we can obtain the following.

Lemma 3. $x(t) \in P C[J, E] \cap X$ is a solution of impulsive $S B V P(1)$ if and only if $x \in P C[J, E] \cap X$ is a solution of the integral equation:

$$
\mu x(t)=\int_{J} G^{*}(t, s) f(s, x(s)) d s,
$$

where

$$
G^{*}(t, s)=\frac{\prod_{0<t_{i}<t}\left(1+\alpha_{i}\right)}{\prod_{0<t_{i}<s}\left(1+\alpha_{i}\right)} G(t, s) .
$$

Let $\alpha(\cdot)$ and $\alpha_{p c}(\cdot)$ donated the Kuratowski noncompactness measure of bounded sets in $E$ and $P C[J, E]$, respectively. If nontrivial function $x \in P C[J, E]$ satisfies problem (5) for some $\mu \neq 0$, then $\mu$ is called an eigenvalue and $x$ is called an eigenfunction of problem (1) corresponding to the eigenvalue $\mu$.

It is well known that the following conclusions hold.

Lemma 4 (see [17]). Let $P$ be a cone in Banach space $E, P_{r}=$ $\{x \in P:\|x\|<r\}(r>0)$, and $\bar{P}_{r}=\{x \in P:\|x\| \leq r\}$. Let operator $A: \bar{P}_{r} \rightarrow P$ be a strict set contraction. If $\|A x\| \geq\|x\|$ and $A x \neq x$ for $x \in \partial P_{r}$, then $i\left(A, P_{r}, P\right)=0$.

Lemma 5 (see [17]). $H \subset C[J, E]$ is relatively compact if and only if $H$ is equicontinuous and for any $t \in J, H(t)$ is a relatively compact set in $E$.

\section{Main Results}

For convenience, let

$$
\begin{gathered}
\sigma_{*}=: \min _{1 \leq j \leq k} \prod_{1 \leq i \leq j}\left(1+\alpha_{i}\right), \quad \sigma^{*}=: \max _{1 \leq j \leq k} \prod_{1 \leq i \leq j}\left(1+\alpha_{i}\right), \\
\Omega(t)=\frac{\varrho(t) \prod_{0<t_{i}<t}\left(1+\alpha_{i}\right)}{\sigma^{*}}, \\
\varrho(t)=\min \left\{\frac{b+a t}{b+a}, \frac{d+c(1-t)}{d+c}\right\}, \quad \forall t \in(0,1) .
\end{gathered}
$$

For the forthcoming analysis, we list the following assumptions:

$$
\begin{aligned}
& \left(H_{1}\right) f \in C[(0,1) \times P \backslash\{\theta\}, P], \\
& \quad\|f(t, x)\| \leq g(t)\|l(x)\|, \quad t \in(0,1), x \in P \backslash\{\theta\},
\end{aligned}
$$

where $g:(0,1) \rightarrow(0,+\infty), l \in C[P \backslash\{\theta\}, P]$ satisfy

$\int_{0}^{1} G(s, s) g(s) l[\Omega(s) r, R] d s<+\infty, \quad \forall R>r>0$,

$$
\text { where } l[r, R]=\sup _{x \in \bar{P}_{R} \backslash P_{r}}\|l(x)\|<+\infty .
$$


$\left(H_{2}\right)$ There exists a constant $L \geq 0$ such that

$$
\begin{array}{r}
\alpha(f(t, D)) \leq L \alpha(D), \quad \forall t \in(0,1), \\
D \subset \bar{P}_{R} \backslash P_{r}, \quad \forall R>r>0, \\
L<\frac{\sigma_{*}}{2 M_{0} \sigma^{*}}, \quad M_{0}=\max _{s \in J} \gamma^{-1}(a s+b)(c(1-s)+d)>0 .
\end{array}
$$

$\left(H_{3}\right)$ There exist $u_{0} \in P \backslash\{\theta\},\left[a_{0}, b_{0}\right] \subset(0,1)$ such that

$$
\begin{gathered}
f(t, x) \geq h(\|x\|) u_{0}, \quad t \in J_{0}=\left[a_{0}, b_{0}\right] \\
\left\|u_{0}\right\| \sigma_{*} \max _{t \in J} \int_{a_{0}}^{b_{0}} G(t, s) \Omega(s) d s \\
\quad<\liminf _{t \rightarrow 0+} \frac{h(t)}{t} \leq+\infty
\end{gathered}
$$

where $h \in C\left[R^{+}, R^{+}\right]$.

$\left(H_{4}\right)$ There exist $\phi \in P^{*}$ and $\left[a_{1}, b_{1}\right] \subset(0,1)$ such that $\phi(x)>0$ for $x>\theta$ and

$$
\liminf _{\|x\| \rightarrow \infty, x \in P} \frac{\phi(f(t, x))}{\phi(x)}=\xi(t)
$$

uniformly for $t \in J_{1}=\left[a_{1}, b_{1}\right]$, and $\int_{J_{1}} G(t, s) \xi(s) d s>$ $\sigma^{*} / \sigma_{*}$.

$\left(H_{5}\right)$ There exist $\phi \in P^{*}$ and $\left[a_{1}, b_{1}\right] \subset(0,1)$ such that $\phi(x)>0$ for $x>\theta$ and

$$
\liminf _{\|x\| \rightarrow 0, x \in P} \frac{\phi(f(t, x))}{\phi(x)}=\xi(t)
$$

uniformly for $t \in J_{1}=\left[a_{1}, b_{1}\right]$, and $\int_{J_{1}} G(t, s) \xi(s) d s>$ $\sigma^{*} / \sigma_{*}$.

Define

$$
Q=\{x \in P C[J, P]: x(t) \geq \Omega(t) x(s), \forall t, s \in J\}
$$

It is easy to check that $Q$ is a cone in space $P C[J, E]$ and $Q \subset$ $P C[J, P]$. Let $Q_{r}=\{x \in Q:\|x\|<r\}$. As in [2], we can prove that

$$
G(t, \tau) \geq \varrho(t) G(s, \tau), \quad \forall t, s, \tau \in[0,1],
$$

where $G(t, s)$ is defined in Lemma 2. So for all $t, s, \tau \in[0,1]$, we have

$$
\begin{aligned}
& G^{*}(t, s) \\
& \quad \geq \frac{\varrho(t) \prod_{0<t_{i}<t}\left(1+\alpha_{i}\right)}{\prod_{0<t_{i}<\tau}\left(1+\alpha_{i}\right)} \cdot \frac{\prod_{0<t_{i}<\tau}\left(1+\alpha_{i}\right)}{\prod_{0<t_{i}<s}\left(1+\alpha_{i}\right)} G(\tau, s) \\
& \quad \geq \Omega(t) G^{*}(\tau, s) .
\end{aligned}
$$

To solve eigenvalues of impulsive SBVP (1), we first consider operator $A$ associated with (5) and defined by

$$
(A x)(t)=\int_{J} G^{*}(t, s) f(s, x(s)) d s, \quad \forall x \in Q \backslash\{\theta\} .
$$

For $\forall x \in Q \backslash\{\theta\}$, from the definition of $Q$, we have

$$
\Omega(t)\|x\|_{p c} \leq\|x(t)\| \leq\|x\|_{p c}, \quad t \in J
$$

By $\left(H_{1}\right)$, we know

$$
\begin{aligned}
& \|(A x)(t)\| \\
& \leq \prod_{0<t_{i}<t}\left(1+\alpha_{i}\right) \int_{0}^{1} \frac{1}{\prod_{0<t_{i}<s}\left(1+\alpha_{i}\right)} G(s, s)\|f(s, x(s))\| d s \\
& \leq \prod_{0<t_{i}<t}\left(1+\alpha_{i}\right) \int_{0}^{1} \frac{1}{\prod_{0<t_{i}<s}\left(1+\alpha_{i}\right)} \\
& \quad \times G(s, s) g(s)\|l(x(s))\| d s \\
& \leq \frac{\sigma^{*}}{\sigma_{*}} \int_{0}^{1} G(s, s) g(s) l\left[\Omega(s)\|x\|_{p c},\|x\|_{p c}\right] d s<+\infty .
\end{aligned}
$$

So the operator $A$ is well defined in $Q \backslash\{\theta\}$.

For the sake of overcoming the singularity, choose $e \epsilon$ int $P$ with $\|e\|=1$ and consider the approximate problem of (17):

$$
\left(A_{m} x\right)(t)=\int_{0}^{1} G^{*}(t, s) f\left(s, x(s)+\frac{e}{m}\right) d s .
$$

For any $x \in Q, t, \tau \in J$,

$$
\begin{aligned}
\left(A_{m} x\right)(t) & =\int_{0}^{1} G^{*}(t, s) f\left(s, x(s)+\frac{e}{m}\right) d s \\
& \geq \Omega(t) \int_{0}^{1} G^{*}(\tau, s) f\left(s, x(s)+\frac{e}{m}\right) d s \\
& =\Omega(t)\left(A_{m} x\right)(\tau) .
\end{aligned}
$$

Hence $A_{m} Q \subset Q$.

Lemma 6. Let conditions $\left(H_{1}\right)$ and $\left(H_{2}\right)$ be satisfied; then for any $r>0$, the operator $A_{m}$ is a strict set contraction from $Q$ into $\mathrm{Q}$. 
Proof. Obviously $A_{m} Q \subset Q$. Now we prove that $A_{m}$ is continuous. Let $\left\|x_{n}-x\right\|_{p c} \rightarrow 0$ as $n \rightarrow \infty\left(x_{n}, x \in \bar{Q}_{r}\right)$; we have

$$
\begin{aligned}
& \left\|\left(A_{m} x_{n}\right)(t)-\left(A_{m} x\right)(t)\right\| \\
& \leq \prod_{0<t_{i}<t}\left(1+\alpha_{i}\right) \int_{0}^{1} \frac{1}{\prod_{0<t_{i}<s}\left(1+\alpha_{i}\right)} G(t, s) \\
& \times \| f\left(s, x_{n}(s)+\frac{e}{m}\right) \\
& -f\left(s, x(s)+\frac{e}{m}\right) \| d s \\
& \leq \prod_{0<t_{i}<t}\left(1+\alpha_{i}\right) \int_{0}^{1} \frac{1}{\prod_{0<t_{i}<s}\left(1+\alpha_{i}\right)} G(s, s) g(s) \\
& \times\left(\left\|l\left(x_{n}(s)+\frac{e}{m}\right)\right\|\right. \\
& \left.+\left\|l\left(x(s)+\frac{e}{m}\right)\right\|\right) \\
& \leq 2 \prod_{0<t_{i}<t}\left(1+\alpha_{i}\right) \int_{0}^{1} \frac{1}{\prod_{0<t_{i}<s}\left(1+\alpha_{i}\right)} G(s, s) g(s) \\
& \times l[\Omega(s) r, r+1] d s \\
& \leq 2 \frac{\sigma^{*}}{\sigma_{*}} \int_{0}^{1} G(s, s) g(s) l[\Omega(s) r, r+1] d s .
\end{aligned}
$$

So $\left(H_{1}\right)$ and the dominated convergence theorem imply that

$$
\lim _{n \rightarrow \infty}\left(A_{m} x_{n}\right)(t)=\left(A_{m} x\right)(t) .
$$

We now show that

$$
\lim _{n \rightarrow \infty}\left\|A_{m} x_{n}-A_{m} x\right\|_{p c}=0 .
$$

In fact, if (24) is not true, then there exist a positive number $\varepsilon_{0}$ and a sequence $\left\{x_{n_{i}}\right\} \subset\left\{x_{n}\right\}$ such that

$$
\left\|A_{m} x_{n_{i}}-A_{m} x\right\|_{p c} \geq \varepsilon_{0} \quad(i=1,2, \ldots) .
$$

Since $\left\{A_{m} x_{n}\right\}$ is relatively compact, there is a subsequence of $\left\{A_{m} x_{n_{i}}\right\}$ which converges to some $x_{0} \in Q$. Without loss of generality, we may assume that $\left\{A_{m} x_{n_{i}}\right\}$ itself converges to $x_{0}$; that is,

$$
\lim _{i \rightarrow \infty}\left\|A_{m} x_{n_{i}}-x_{0}\right\|_{p c}=0 .
$$

By virtue of (23) and (26), we have $x_{0}=A_{m} x$, and so (26) contradicts with (25). Hence (24) holds, and the continuity of $A_{m}$ is proved. Q.

By $\left(H_{1}\right)$, it is easy to see that $A_{m}$ is bounded from $Q$ into

Now we will prove that the operator $A_{m}$ is a strict set contraction from $Q$ into $Q$. Let

$$
\left(A_{m}^{(n)} x\right)(t)=\int_{1 / n}^{1-(1 / n)} G^{*}(t, s) f\left(s, x(s)+\frac{e}{m}\right) d s .
$$

By $\left(H_{1}\right)$, as $n \rightarrow \infty$, we have

$$
\begin{aligned}
& \left\|\left(A_{m} x\right)(t)-\left(A_{m}^{(n)} x\right)(t)\right\| \\
& \quad \leq \int_{0}^{1 / n} G^{*}(t, s)\left\|f\left(s, x(s)+\frac{e}{m}\right)\right\| d s \\
& \quad+\int_{1-(1 / n)}^{1} G^{*}(t, s)\left\|f\left(s, x(s)+\frac{e}{m}\right)\right\| d s \longrightarrow 0 .
\end{aligned}
$$

So for any bounded set $U \subset Q$, we have

$$
\lim _{n \rightarrow \infty}\left(A_{m}^{(n)} x\right)(t)=\left(A_{m} x\right)(t), \quad \forall x \in U, t \in J .
$$

Hence

$$
d_{h}\left(\left(A_{m}^{(n)} U\right)(t),\left(A_{m} U\right)(t)\right) \longrightarrow 0, \quad n \longrightarrow \infty
$$

where $d_{h}(\cdot, \cdot)$ denotes the Hausdorff metric, which implies

$$
\alpha\left(\left(A_{m}^{(n)} U\right)(t)\right) \longrightarrow \alpha\left(\left(A_{m} U\right)(t)\right), \quad n \longrightarrow \infty, t \in J
$$

For any $t \in J$, by $\left(H_{2}\right)$, we have

$$
\begin{aligned}
& \alpha\left(\left(A_{m}^{(n)} U\right)(t)\right) \\
& =\alpha\left(\left\{\prod_{0<t_{i}<t}\left(1+\alpha_{i}\right)\right.\right. \\
& \quad \times \int_{1 / n}^{1-(1 / n)} \frac{1}{\prod_{0<t_{i}<s}\left(1+\alpha_{i}\right)} G(t, s) \\
& \left.\left.\quad \times f\left(s, x(s)+\frac{e}{m}\right) d s, x \in U\right\}\right) \\
& \leq\left(1-\frac{1}{2 n}\right) \prod_{0<t_{i}<t}\left(1+\alpha_{i}\right) \\
& \times \alpha\left(\frac { 1 } { c o } \left\{\frac{1}{\prod_{0<t_{i}<s}\left(1+\alpha_{i}\right)} G(t, s)\right.\right. \\
& \left.\left.\quad \times f\left(s, x(s)+\frac{e}{m}\right): s \in\left[\frac{1}{n}, 1-\frac{1}{n}\right], x \in U\right\}\right)
\end{aligned}
$$




$$
\begin{aligned}
& \leq \prod_{0<t_{i}<t}\left(1+\alpha_{i}\right) \\
& \quad \times \alpha\left(\left\{\frac{1}{\prod_{0<t_{i}<s}\left(1+\alpha_{i}\right)} G(s, s)\right.\right. \\
& \left.\left.\quad \times f\left(s, x(s)+\frac{e}{m}\right): s \in\left[\frac{1}{n}, 1-\frac{1}{n}\right], x \in U\right\}\right) \\
& \leq \frac{\sigma^{*}}{\sigma_{*}} M_{0} \alpha\left(f\left(s, U\left(I_{n}\right)\right)\right), \quad I_{n}=\left[\frac{1}{n}, 1-\frac{1}{n}\right], s \in I_{n}, \\
& \leq L M_{0} \frac{\sigma^{*}}{\sigma_{*}} \alpha\left(U\left(I_{n}\right)\right) \\
& \leq 2 L M_{0} \frac{\sigma^{*}}{\sigma_{*}} \alpha_{p c}(U) \\
& <\alpha_{p c}(U) .
\end{aligned}
$$

It is easy to show that $\alpha_{p c}\left(A_{m}(U)\right)=\sup _{t \in J} \alpha\left(\left(A_{m}(U)\right)(t)\right)$. Therefore, we can have $\alpha_{p c}\left(A_{m} U\right) \leq 2 L M_{0}\left(\sigma^{*} / \sigma_{*}\right) \alpha_{p c}(U)<$ $\alpha_{p c}(U)$. Consequently, the operator $A_{m}$ is a strict set contraction from $Q$ into $Q$. The proof is thus completed.

Theorem 7. Let conditions $\left(H_{1}\right),\left(H_{2}\right)$, and $\left(H_{3}\right)$ be satisfied; then there exists a positive number $\delta$ such that for any given $r \in$ $(0, \delta)$, the impulsive SBVP (1) has a nontrivial eigenfunction $x_{r} \in P C[J, P]$ with $\left\|x_{r}\right\|_{p c}=r$ corresponding to eigenvalue $\mu_{r} \geq 1$.

Proof. By Lemma 6, the operator $A_{m}$ is a strict set contraction from $\bar{Q}_{r}$ into $Q$. Observing $\left(H_{3}\right)$, if $\liminf _{t \rightarrow 0+}(h(t) / t) \neq+\infty$, we can choose a $v_{0}$ with

$$
\frac{1}{2} \liminf _{t \rightarrow 0+} \frac{h(t)}{t} \leq v_{0}<\liminf _{t \rightarrow 0+} \frac{h(t)}{t},
$$

and there is a $\delta>0$ such that

$$
h(t) \geq v_{0} t, \quad \forall 0<t<\delta .
$$

Choose $r \in(0, \delta)$ and $m$ sufficiently large such that $(1 / m)<$ $\delta-r$; we have

$$
\begin{aligned}
& \left(A_{m} x\right)(t) \\
& =\prod_{0<t_{i}<t}\left(1+\alpha_{i}\right) \int_{0}^{1} \frac{1}{\prod_{0<t_{i}<s}\left(1+\alpha_{i}\right)} \\
& \quad \times G(t, s) f\left(s, x(s)+\frac{e}{m}\right) d s \\
& \geq \prod_{0<t_{i}<t}\left(1+\alpha_{i}\right) \int_{a_{0}}^{b_{0}} \frac{1}{\prod_{0<t_{i}<s}\left(1+\alpha_{i}\right)} G(t, s)
\end{aligned}
$$

$$
\begin{array}{r}
\times h\left(\left\|x(s)+\frac{e}{m}\right\|\right) u_{0} d s \\
\geq \prod_{0<t_{i}<t}\left(1+\alpha_{i}\right) \int_{a_{0}}^{b_{0}} \frac{1}{\prod_{0<t_{i}<s}\left(1+\alpha_{i}\right)} \\
\times G(t, s) v_{0}\|x(s)\| u_{0} d s \\
\geq \frac{\sigma_{*}}{\sigma^{*}} \int_{a_{0}}^{b_{0}} G(t, s) v_{0} \Omega(s)\|x\|_{P C} u_{0} d s .
\end{array}
$$

So

$$
\begin{aligned}
& \left\|A_{m} x\right\|_{P C} \\
& \quad \geq \frac{\sigma_{*}}{\sigma^{*}} v_{0}\left\|u_{0}\right\| \max _{t \in J} \int_{a_{0}}^{b_{0}} G(t, s) \Omega(s) d s\|x\|_{P C}>\|x\|_{p c} .
\end{aligned}
$$

By virtue of Lemma 4 , we have $i\left(A_{m}, Q_{r}, Q\right)=0$. Since $i\left(\theta, Q_{r}, Q\right)=1$, it follows from the homotopy invariance of fixed point index for strict set contraction that there exist $x_{m} \in \partial \bar{Q}_{r}$ and $\lambda_{m} \in(0,1)$ such that

$$
x_{m}=\lambda_{m} A_{m} x_{m}, \quad \text { i.e. } A_{m} x_{m}=\lambda_{m}^{-1} x_{m},
$$

where $m \geq m_{0}=[1 /(\delta-r)]+1$.

Let $B=:\left\{x_{m}: m \geq m_{0}\right\}$. Obviously, $B$ is uniformly bounded. We shall show that $B$ is equicontinuous. By virtue of boundary condition, we need to consider only the following eight cases.

Case 1. $b=d=0$, the boundary value condition is $x(0)=$ $x(1)=0$. So we need only to show that $\left\{x_{m}(t)\right\}$ uniformly converge to $\theta$ with respect to $m \geq m_{0}$ as $t \rightarrow 0^{+}, t \rightarrow 1-0$, and $B$ is equicontinuous on any closed subinterval of $(0,1)$.

By $\left(H_{1}\right)$, let

$$
\eta=: \int_{0}^{1} G(s, s) g(s) l\left[\Omega(s) r, r+\frac{1}{m_{0}}\right] d s<+\infty .
$$

Using the absolute continuity of integration, for any $\varepsilon>0$, there exists a $\delta_{1}>0$, such that

$$
\int_{t_{1}}^{t_{2}} G(s, s) g(s) l\left[\Omega(s) r, r+\frac{1}{m_{0}}\right] d s<\varepsilon
$$

as $\left|t_{1}-t_{2}\right|<\delta_{1}, t_{1}, t_{2} \in J$. Since

$$
\begin{aligned}
x_{m}(t)=\lambda_{m} \int_{0}^{1} G^{*}(t, s) f\left(s, x_{m}(s)+\frac{e}{m}\right) d s \\
\leq \lambda_{m} \frac{\sigma^{*}}{\sigma_{*}}\left[(1-t) \int_{0}^{t} s f\left(s, x_{m}(s)+\frac{e}{m}\right) d s\right. \\
\left.\quad+t \int_{t}^{1}(1-s) f\left(s, x_{m}(s)+\frac{e}{m}\right) d s\right],
\end{aligned}
$$


we have

$$
\begin{aligned}
& \left\|x_{m}(t)\right\| \\
& \leq \frac{\sigma^{*}}{\sigma_{*}}\left\{(1-t) \int_{0}^{t} s g(s)\left\|l\left(x_{m}(s)+\frac{e}{m}\right)\right\| d s\right. \\
& \left.\quad+t \int_{t}^{1}(1-s) g(s)\left\|l\left(x_{m}(s)+\frac{e}{m}\right)\right\| d s\right\} \\
& \leq \frac{\sigma^{*}}{\sigma_{*}}\left\{(1-t) \int_{0}^{t} s g(s) l\left[\Omega(s) r, r+\frac{1}{m_{0}}\right] d s\right. \\
& \left.+t \int_{t}^{1}(1-s) g(s) l\left[\Omega(s) r, r+\frac{1}{m_{0}}\right] d s\right\} .
\end{aligned}
$$

To prove that $\left\{x_{m}(t)\right\}$ uniformly converge to $\theta$ with respect to $m \geq m_{0}$ as $t \rightarrow 0^{+}$, we need only to show

$$
\begin{aligned}
& \lim _{t \rightarrow 0^{+}}(1-t) \int_{0}^{t} s g(s) l\left[\Omega(s) r, r+\frac{1}{m_{0}}\right] d s=0, \\
& \lim _{t \rightarrow 0^{+}} t \int_{t}^{1}(1-s) g(s) l\left[\Omega(s) r, r+\frac{1}{m_{0}}\right] d s=0 .
\end{aligned}
$$

Notice that

$$
\begin{aligned}
& (1-t) \int_{0}^{t} s g(s) l\left[\Omega(s) r, r+\frac{1}{m_{0}}\right] d s \\
& \leq \int_{0}^{t}(1-s) s g(s) l\left[\Omega(s) r, r+\frac{1}{m_{0}}\right] d s \\
& =\int_{0}^{t} G(s, s) g(s) l\left[\Omega(s) r, r+\frac{1}{m_{0}}\right] d s .
\end{aligned}
$$

This together with (39) implies that (42) holds. For $\varepsilon, \delta_{1}$ in (39), choose

$$
\delta_{2}=\min \left\{\delta_{1}, \frac{\varepsilon \delta_{1}}{\eta}\right\}
$$

Then for $t \in\left(0, \delta_{2}\right)$, (39) implies that

$$
\begin{aligned}
& t \int_{t}^{1}(1-s) g(s) l\left[\Omega(s) r, r+\frac{1}{m_{0}}\right] d s \\
& \quad \leq t \int_{t}^{\delta_{1}}(1-s) g(s) l\left[\Omega(s) r, r+\frac{1}{m_{0}}\right] d s
\end{aligned}
$$

$$
\begin{aligned}
& +\int_{\delta_{1}}^{1} \frac{t}{s} s(1-s) g(s) l\left[\Omega(s) r, r+\frac{1}{m_{0}}\right] d s \\
\leq & \int_{t}^{\delta_{1}} s(1-s) g(s) l\left[\Omega(s) r, r+\frac{1}{m_{0}}\right] d s \\
& +\frac{t}{\delta_{1}} \int_{\delta_{1}}^{1} s(1-s) g(s) l\left[\Omega(s) r, r+\frac{1}{m_{0}}\right] d s \\
= & \int_{t}^{\delta_{1}} G(s, s) g(s) l\left[\Omega(s) r, r+\frac{1}{m_{0}}\right] d s \\
& +\frac{t}{\delta_{1}} \int_{\delta_{1}}^{1} G(s, s) g(s) l\left[\Omega(s) r, r+\frac{1}{m_{0}}\right] d s \\
\leq & \varepsilon+\frac{t}{\delta_{1}} \int_{0}^{1} G(s, s) g(s) l\left[\Omega(s) r, r+\frac{1}{m_{0}}\right] d s \\
\leq & 2 \varepsilon .
\end{aligned}
$$

Hence (43) holds. Very similarly, we can obtain that $\left\{x_{m}(t)\right\}$ uniformly converge to $\theta$ with respect to $m \geq m_{0}$ as $t \rightarrow 1-0$. Now, we show that $B$ is equicontinuous on $[\zeta, 1-\zeta]$ for any $[\zeta, 1-\zeta] \subset(0,1), \zeta \in(0,1 / 2)$. Notice that

$$
\begin{aligned}
& x_{m}\left(t_{2}\right)-x_{m}\left(t_{1}\right) \lambda_{m} \prod_{0<t_{i}<t}\left(1+\alpha_{i}\right) \\
& \times {\left[\left(1-t_{2}\right) \int_{0}^{t_{2}} s \frac{1}{\prod_{0<t_{i}<s}\left(1+\alpha_{i}\right)} f\left(s, x_{m}(s)+\frac{e}{m}\right) d s\right.} \\
&+t_{2} \int_{t_{2}}^{1}(1-s) \frac{1}{\prod_{0<t_{i}<s}\left(1+\alpha_{i}\right)} f\left(s, x_{m}(s)+\frac{e}{m}\right) d s \\
&-\left(1-t_{1}\right) \int_{0}^{t_{1}} s \frac{1}{\prod_{0<t_{i}<s}\left(1+\alpha_{i}\right)} f\left(s, x_{m}(s)+\frac{e}{m}\right) d s \\
&\left.-t_{1} \int_{t_{1}}^{1}(1-s) \frac{1}{\prod_{0<t_{i}<s}\left(1+\alpha_{i}\right)} f\left(s, x_{m}(s)+\frac{e}{m}\right) d s\right] \\
& \prod_{0<t_{i}<t}\left(1+\alpha_{i}\right) \\
& \times {\left[\left(t_{2}-t_{1}\right)\right.} \\
& \times\left(-\int_{t_{2}}^{1} s \frac{1}{\prod_{0<t_{i}<s}\left(1+\alpha_{i}\right)} f\left(s, x_{m}(s)+\frac{e}{m}\right) d s\right. \\
& \prod_{0<t_{i}<s}\left(1+\alpha_{i}\right) \\
& t_{2}
\end{aligned}
$$


Journal of Function Spaces

7

$$
\begin{aligned}
&+\left(1-t_{1}\right) \int_{t_{1}}^{t_{2}} s \frac{1}{\prod_{0<t_{i}<s}\left(1+\alpha_{i}\right)} \\
& \times f\left(s, x_{m}(s)+\frac{e}{m}\right) d s \\
&-t_{1} \int_{t_{1}}^{t_{2}}(1-s) \frac{1}{\prod_{0<t_{i}<s}\left(1+\alpha_{i}\right)} \\
&\left.\times f\left(s, x_{m}(s)+\frac{e}{m}\right) d s\right] .
\end{aligned}
$$

So for any $t_{1}, t_{2} \in[\zeta, 1-\zeta], t_{2}>t_{1}, m \geq m_{0}$, we have

$$
\begin{aligned}
& \left\|x_{m}\left(t_{2}\right)-x_{m}\left(t_{1}\right)\right\| \\
& \leq \prod_{0<t_{i}<t}\left(1+\alpha_{i}\right) \\
& \times\left\{( t _ { 2 } - t _ { 1 } ) \left[\int_{0}^{t_{2}} s \frac{1}{\prod_{0<t_{i}<s}\left(1+\alpha_{i}\right)}\right.\right. \\
& \times g(s)\left\|l\left(x_{m}(s)+\frac{e}{m}\right)\right\| d s \\
& +\int_{t_{2}}^{1}(1-s) \frac{1}{\prod_{0<t_{i}<s}\left(1+\alpha_{i}\right)} \\
& +\left(1-t_{1}\right) \int_{t_{1}}^{t_{2}} s \frac{1}{\prod_{0<t_{i}<s}\left(1+\alpha_{i}\right)} \\
& \times g(s)\left\|l\left(x_{m}(s)+\frac{e}{m}\right)\right\| d s \\
& +\frac{1}{t_{2}} \int_{t_{2}}^{1} s(1-s) \overline{\left.\left.x_{m}(s)+\frac{e}{m}\right) \| d s\right]} \\
& \times \prod_{t_{1}}^{t_{2}}(1-s) \frac{1}{\prod_{0<t_{i}<s}\left(1+\alpha_{i}\right)} \\
& \left.\times g(s)\left\|l\left(x_{m}(s)+\frac{e}{m}\right)\right\| d s\right\} \\
& \times\left\{t_{i}-t_{1}\right)\left(\frac{1}{1-t_{2}} \int_{0}^{t_{2}}(1-s) s \frac{1}{\prod_{0<t_{i}<s}\left(1+\alpha_{i}\right)}\right. \\
& \times g(s) l\left[\Omega(s) r, r+\frac{1}{m_{0}}\right] d s
\end{aligned}
$$

$$
\begin{aligned}
& +\frac{1-t_{1}}{1-t_{2}} \int_{t_{1}}^{t_{2}} s(1-s) \frac{1}{\prod_{0<t_{i}<s}\left(1+\alpha_{i}\right)} \\
& \times g(s) l\left[\Omega(s) r, r+\frac{1}{m_{0}}\right] d s \\
& +\int_{t_{1}}^{t_{2}} s(1-s) \frac{1}{\prod_{0<t_{i}<s}\left(1+\alpha_{i}\right)} \\
& \left.\times g(s) l\left[\Omega(s) r, r+\frac{1}{m_{0}}\right] d s\right\} \\
& \leq \prod_{0<t_{i}<t}\left(1+\alpha_{i}\right) \\
& \times\left\{( t _ { 2 } - t _ { 1 } ) \left(\frac{1}{1-t_{2}} \int_{0}^{t_{2}} G(s, s) \frac{1}{\prod_{0<t_{i}<s}\left(1+\alpha_{i}\right)}\right.\right. \\
& \times g(s) l\left[\Omega(s) r, r+\frac{1}{m_{0}}\right] d s \\
& +\frac{1}{t_{2}} \int_{t_{2}}^{1} G(s, s) \frac{1}{\prod_{0<t_{i}<s}\left(1+\alpha_{i}\right)} \\
& \left.\times g(s) l\left[\Omega(s) r, r+\frac{1}{m_{0}}\right] d s\right) \\
& +\frac{1-t_{1}}{1-t_{2}} \int_{t_{1}}^{t_{2}} G(s, s) \frac{1}{\prod_{0<t_{i}<s}\left(1+\alpha_{i}\right)} \\
& \times g(s) l\left[\Omega(s) r, r+\frac{1}{m_{0}}\right] d s \\
& +\int_{t_{1}}^{t_{2}} G(s, s) \frac{1}{\prod_{0<t_{i}<s}\left(1+\alpha_{i}\right)} \\
& \left.\times g(s) l\left[\Omega(s) r, r+\frac{1}{m_{0}}\right] d s\right\} \\
& \leq \prod_{0<t_{i}<t}\left(1+\alpha_{i}\right) \\
& \times\left\{( t _ { 2 } - t _ { 1 } ) \left(\frac{1}{\zeta} \int_{0}^{t_{2}} G(s, s) \frac{1}{\prod_{0<t_{i}<s}\left(1+\alpha_{i}\right)}\right.\right. \\
& \times g(s) l\left[\Omega(s) r, r+\frac{1}{m_{0}}\right] d s \\
& +\frac{1}{\zeta} \int_{t_{2}}^{1} G(s, s) \frac{1}{\prod_{0<t_{i}<s}\left(1+\alpha_{i}\right)} \\
& \left.\times g(s) l\left[\Omega(s) r, r+\frac{1}{m_{0}}\right]\right) \\
& +\left(\frac{1-\zeta}{\zeta}+1\right) \int_{t_{1}}^{t_{2}} G(s, s) \frac{1}{\prod_{0<t_{i}<s}\left(1+\alpha_{i}\right)} \\
& \left.\times g(s) l\left[\Omega(s) r, r+\frac{1}{m_{0}}\right] d s\right\}
\end{aligned}
$$




$$
\begin{aligned}
& \leq \frac{\sigma^{*}}{\sigma_{*}}\left\{\left(t_{2}-t_{1}\right) \frac{2 \eta}{\zeta}\right. \\
& \left.\quad+\frac{1}{\zeta} \int_{t_{1}}^{t_{2}} G(s, s) g(s) l\left[\Omega(s) r, r+\frac{1}{m_{0}}\right] d s\right\} .
\end{aligned}
$$

This together with (39) implies that $B$ is equicontinuous on $J$.

Case 2. $b=0, d \neq 0, c \neq 0$, the boundary value condition is $x(0)=0, c x(1)+d x^{\prime}(1)=0$. We need to show that $\left\{x_{m}(t)\right\}$ uniformly converge to $\theta$ with respect to $m \geq m_{0}$ as $t \rightarrow 0^{+}$. This can be obtained by the similar way in Case 1 , so we omit it. Next, we show $B$ is equicontinuous on $[\iota, 1]$ for any $\iota \in(0,1)$. In fact, for any $t_{1}, t_{2} \in[\iota, 1], t_{2}>t_{1}, m \geq m_{0}$, we have

$$
\begin{aligned}
& \left\|x_{m}\left(t_{2}\right)-x_{m}\left(t_{1}\right)\right\| \\
& \leq \int_{0}^{t_{1}}\left|G^{*}\left(t_{2}, s\right)-G^{*}\left(t_{1}, s\right)\right|\left\|f\left(s, x_{m}(s)+\frac{e}{m}\right)\right\| d s \\
& +\int_{t_{1}}^{t_{2}}\left|G^{*}\left(t_{2}, s\right)-G^{*}\left(t_{1}, s\right)\right|\left\|f\left(s, x_{m}(s)+\frac{e}{m}\right)\right\| d s \\
& +\int_{t_{2}}^{1}\left|G^{*}\left(t_{2}, s\right)-G^{*}\left(t_{1}, s\right)\right|\left\|f\left(s, x_{m}(s)+\frac{e}{m}\right)\right\| d s \\
& \leq\left(t_{2}-t_{1}\right) \frac{c}{c(1-s)+d} \frac{\sigma^{*}}{\sigma_{*}} \\
& \times \int_{0}^{t_{1}} \frac{s(c(1-s)+d)}{c+d} g(s)\left\|l\left(x_{m}(s)+\frac{e}{m}\right)\right\| d s \\
& +2 \frac{\sigma^{*}}{\sigma_{*}} \int_{t_{1}}^{t_{2}} G(s, s) g(s)\left\|l\left(x_{m}(s)+\frac{e}{m}\right)\right\| d s \\
& +\left(t_{2}-t_{1}\right) \frac{1}{s} \frac{\sigma^{*}}{\sigma_{*}} \\
& \times \int_{t_{2}}^{1} \frac{s(c(1-s)+d)}{c+d} g(s)\left\|l\left(x_{m}(s)+\frac{e}{m}\right)\right\| d s \\
& \leq\left(t_{2}-t_{1}\right) \frac{c}{d} \frac{\sigma^{*}}{\sigma_{*}} \int_{0}^{t_{1}} G(s, s) g(s) l\left[\Omega(s) r, r+\frac{1}{m_{0}}\right] d s \\
& +2 \frac{\sigma^{*}}{\sigma_{*}} \int_{t_{1}}^{t_{2}} G(s, s) g(s) l\left[\Omega(s) r, r+\frac{1}{m_{0}}\right] d s \\
& +\left(t_{2}-t_{1}\right) \frac{1}{\iota} \frac{\sigma^{*}}{\sigma_{*}} \int_{t_{2}}^{1} G(s, s) g(s) l\left[\Omega(s) r, r+\frac{1}{m_{0}}\right] d s \\
& \leq\left(t_{2}-t_{1}\right) \eta \frac{\sigma^{*}}{\sigma_{*}}\left(\frac{c}{d}+\frac{1}{l}\right) \\
& +2 \frac{\sigma^{*}}{\sigma_{*}} \int_{t_{1}}^{t_{2}} G(s, s) g(s) l\left[\Omega(s) r, r+\frac{1}{m_{0}}\right] d s .
\end{aligned}
$$

This together with (39) implies that $B$ is equicontinuous on $J$.
Case 3. $b=0, d \neq 0, c=0$, the boundary value condition is $x(0)=0, x^{\prime}(1)=0$. The equicontinuity of $B$ on $J$ of this case can be obtained by the same way of Case 2 , so we omit it.

Case 4. $d=0, b \neq 0, a \neq 0$, the boundary value condition is $a x(0)-b x^{\prime}(0)=0, x(1)=0$. Similarly, by Case 1 , we can get that $\left\{x_{m}(t)\right\}$ uniformly converge to $\theta$ with respect to $m \geq m_{0}$ as $t \rightarrow 1^{-}$. For any $\varsigma \in(0,1)$, we need to show that $B$ is equicontinuous on $[0, \varsigma]$. In fact, for any $t_{1}, t_{2} \in[0, \varsigma], t_{2}>$ $t_{1}, m \geq m_{0}$, we have

$$
\begin{aligned}
\| x_{m} & \left(t_{2}\right)-x_{m}\left(t_{1}\right) \| \\
\leq & \int_{0}^{t_{1}}\left|G^{*}\left(t_{2}, s\right)-G^{*}\left(t_{1}, s\right)\right|\left\|f\left(s, x_{m}(s)+\frac{e}{m}\right)\right\| d s \\
& +\int_{t_{1}}^{t_{2}}\left|G^{*}\left(t_{2}, s\right)-G^{*}\left(t_{1}, s\right)\right|\left\|f\left(s, x_{m}(s)+\frac{e}{m}\right)\right\| d s \\
& +\int_{t_{2}}^{1}\left|G^{*}\left(t_{2}, s\right)-G^{*}\left(t_{1}, s\right)\right|\left\|f\left(s, x_{m}(s)+\frac{e}{m}\right)\right\| d s \\
\leq & \left(t_{2}-t_{1}\right) \frac{1}{1-s} \frac{\sigma^{*}}{\sigma_{*}} \int_{0}^{t_{1}} G(s, s) g(s) l\left[\Omega(s) r, r+\frac{1}{m_{0}}\right] d s \\
& +2 \frac{\sigma^{*}}{\sigma_{*}} \int_{t_{1}}^{t_{2}} G(s, s) g(s) l\left[\Omega(s) r, r+\frac{1}{m_{0}}\right] d s \\
& +\left(t_{2}-t_{1}\right) \frac{a}{a s+b} \frac{\sigma^{*}}{\sigma_{*}} \int_{t_{2}}^{1} G(s, s) g(s) \\
& +2 \frac{\sigma^{*}}{\sigma_{*}} \int_{t_{1}}^{t_{2}} G(s, s) g(s) l\left[\Omega(s) r, r+\frac{1}{m_{0}}\right] d s . \\
\leq & \left(t_{2}-t_{1}\right) \frac{1}{1-\varsigma} \frac{\sigma^{*}}{\sigma_{*}} \int_{0}^{t_{1}} G(s, s) g(s) l\left[\Omega(s) r, r+\frac{1}{m_{0}}\right] d s \\
& +2 \frac{\sigma^{*}}{\sigma_{*}} \int_{t_{1}}^{t_{2}} G(s, s) g(s) l\left[\Omega(s) r, r+\frac{1}{m_{0}}\right] d s \\
& \left.+t_{1}\right) \frac{b}{a} \frac{\sigma^{*}}{\sigma_{*}} \int_{t_{2}}^{1} G(s, s) g(s) l\left[\Omega(s) r, r+\frac{1}{m_{0}}\right] d s \\
& \\
& \\
& \\
&
\end{aligned}
$$

This together with (39) implies that $B$ is equicontinuous on $J$.

Case 5. $d=0, b \neq 0, a=0$, the boundary value condition is $x^{\prime}(0)=0, x(1)=0$.

Case 6. $a=0, b d \neq 0, c \neq 0$, the boundary value condition is $x^{\prime}(0)=0, c x(1)+d x^{\prime}(1)=0$. 
Case 7. $a \neq 0, b d \neq 0, c=0$, the boundary value condition is $a x(0)-b x^{\prime}(0)=0, x^{\prime}(1)=0$.

Case $8 . \quad b d \neq 0, a c \neq 0$, the boundary value condition is $a x(0)-$ $b x^{\prime}(0)=0, c x(1)+d x^{\prime}(1)=0$.

By the very similar methods in Cases 2 and 4, we can prove that $B$ is equicontinuous on $J$ in Cases $5-8$. Hence, the proofs are omitted. Next we show that $B$ is relatively compact. It is easy to see that

$$
\begin{aligned}
& \alpha(B(t)) \\
& \leq \alpha\left(\left\{\prod_{0<t_{i}<t}\left(1+\alpha_{i}\right) \int_{0}^{1} \frac{1}{\prod_{0<t_{i}<s}\left(1+\alpha_{i}\right)} G(t, s)\right.\right. \\
& \left.\left.\times f\left(s, x_{m}(s)+\frac{e}{m}\right) d s: m \geq m_{0}\right\}\right) \\
& \leq 2 \prod_{0<t_{i}<t}\left(1+\alpha_{i}\right) \int_{0}^{1} \frac{1}{\prod_{0<t_{i}<s}\left(1+\alpha_{i}\right)} G(t, s) \\
& \times \alpha\left(\left\{f\left(s, x_{m}(s)+\frac{e}{m}\right): m \geq m_{0}\right\}\right) d s \\
& \leq 2 \frac{\sigma^{*}}{\sigma_{*}} L \int_{0}^{1} G(t, s) \alpha(B(s)) d s .
\end{aligned}
$$

Hence, we have

$$
\begin{aligned}
\alpha_{p c}(B) & \leq 2 \frac{\sigma^{*}}{\sigma_{*}} L \int_{0}^{1} G(t, s) d s \alpha_{p c}(B) \\
& \leq 2 \frac{\sigma^{*}}{\sigma_{*}} L \int_{0}^{1} G(s, s) d s \alpha_{p c}(B) \\
& \leq 2 L M_{0} \frac{\sigma^{*}}{\sigma_{*}} \alpha_{p c}(B) .
\end{aligned}
$$

Observing $L<\sigma_{*} / 2 M_{0} \sigma^{*}$, so $\alpha_{p c}(B)=0$, which means $B$ is relatively compact.

It follows from Lemma 5 that there is a convergent subsequence of $\left\{x_{m}\right\}$, and without loss of generality, we may assume that $\left\{x_{m}\right\}$ itself converges to some $x_{r} \in \partial \bar{Q}_{r}$, and $\lim _{m \rightarrow \infty} \lambda_{m}=\lambda_{r} \in[0,1]$. Hence (40) and the dominated convergence theorem imply that

$$
x_{r}(t)=\lambda_{r} \int_{0}^{1} G^{*}(t, s) f\left(s, x_{r}(s)\right) d s=\lambda_{r} A x_{r}(t), \quad t \in J .
$$

Obviously, $\lambda_{r}>0$. Consequently, $A x_{r}=\lambda_{r}^{-1} x_{r}=\mu_{r} x_{r}$, where $\mu_{r}=\lambda_{r}^{-1} \geq 1$.

The case $\liminf \operatorname{in}_{t \rightarrow 0+}(h(t) / t)=+\infty$ can be proved similarly, so it is omitted. Then the theorem is proved.

Theorem 8. Let conditions $\left(H_{1}\right),\left(H_{2}\right)$, and $\left(H_{4}\right)$ be satisfied; then there exists a positive number $M$ such that for any given $r>M / \min _{t \in J} \Omega(t)$, the impulsive SBVP (1) has a nontrivial eigenfunction $x_{r} \in P C[J, P]$ with $\left\|x_{r}\right\|_{P C}=r$ corresponding to eigenvalue $\mu_{r} \geq 1$.

Proof. By $\left(H_{4}\right)$, there exist $\varepsilon_{0}>0$ and $M>0$ such that

$$
\int_{a_{1}}^{b_{1}} G(t, s)\left(\xi(s)-\varepsilon_{0}\right) d s>\frac{\sigma^{*}}{\sigma_{*}},
$$

and $\phi(f(t, x)) \geq\left(\xi(t)-\varepsilon_{0}\right) \phi(x)$ for $t \in J_{1}=\left[a_{1}, b_{1}\right], x \in P$ with $\|x\|>M$.

Choose $r>M / \min _{t \in J} \Omega(t)$. If there exists $x_{0} \in \partial \bar{Q}$ such that $x_{0} \geq A_{m} x_{0}$, then

$$
\left\|x_{0}(s)\right\| \geq \Omega(s)\left\|x_{0}\right\|_{p c}=\Omega(s) r>M, \quad s \in J .
$$

Hence, for $t \in J_{1}$, we have

$$
\begin{aligned}
& \phi\left(x_{0}(t)\right) \\
& \geq \prod_{0<t_{i}<t}\left(1+\alpha_{i}\right) \int_{0}^{1} \frac{1}{\prod_{0<t_{i}<s}\left(1+\alpha_{i}\right)} \\
& \quad \times G(t, s) \phi\left(f\left(s, x_{0}(s)+\frac{e}{m}\right)\right) d s \\
& \geq \prod_{0<t_{i}<t}\left(1+\alpha_{i}\right) \int_{J_{1}} \frac{1}{\prod_{0<t_{i}<s}\left(1+\alpha_{i}\right)} \\
& \geq \prod_{0<t_{i}<t}\left(1+\alpha_{i}\right) \int_{J_{1}} \frac{1}{\prod_{0<t_{i}<s}\left(1+\alpha_{i}\right)} \\
& \left.\times \frac{\sigma_{*} \int_{J_{1}} G(t, s)\left(\xi(s)-\varepsilon_{0}\right) \phi\left(x_{0}(s)+\frac{e}{m}\right) d s}{\sigma^{*}}(s)-\varepsilon_{0}\right) \phi\left(x_{0}(s)\right) d s
\end{aligned}
$$

Obviously,

$$
\inf _{t \in J_{1}}\left\|x_{0}(t)\right\| \geq \Omega(t)\left\|x_{0}\right\|_{P C}=\Omega(t) r>0 .
$$

The continuity of $x_{0}(t)$ implies that $\phi\left(x_{0}(t)\right)$ is continuous on $J_{1}$. Hence, this together with $(57)$ and $\left(H_{4}\right)$ implies that $\min _{t \in J_{1}} \phi\left(x_{0}(t)\right)>0$. Observing (54) and (56), we can obtain a contradiction. Applying Lemma 4, we know that the fixed point index $i\left(A_{m}, Q_{r}, Q\right)=0$. Since $i\left(\theta, Q_{r}, Q\right)=1$, it follows from the homotopy invariance of fixed point index for strict set contraction that there exist $x_{m} \in \partial \bar{Q}_{r}$ and $0<\lambda_{m}<1$ such that

$$
x_{m}=\lambda_{m} A_{m} x_{m}, \quad \text { i.e. } A_{m} x_{m}=\lambda_{m}^{-1} x_{m}=\mu_{m} x_{m},
$$


where $\mu_{m}=\lambda_{m}^{-1}>1$. The rest of the proof is completely similar to that of Theorem 7. So it is omitted.

Theorem 9. Let conditions $\left(H_{1}\right),\left(H_{2}\right)$, and $\left(H_{5}\right)$ be satisfied; then there exists a positive number $\delta$ such that for any given $r \in$ $(0, \delta)$, the impulsive SBVP (1) has a nontrivial eigenfunction $x_{r} \in P C[J, P]$ with $\left\|x_{r}\right\|_{P C}=r$ corresponding to eigenvalue $\mu_{r} \geq 1$.

Proof. By $\left(H_{5}\right)$, there exist $\varepsilon_{1}>0$ and $\delta>0$ such that

$$
\int_{J_{1}} G(t, s)\left(\xi(s)-\varepsilon_{1}\right) d s>1
$$

and $\phi(f(t, x)) \geq\left(\xi(t)-\varepsilon_{1}\right) \phi(x)$ for $t \in J_{1}=\left[a_{1}, b_{1}\right], x \in P$ with $\|x\|<\delta$. Choose $r \in(0, \delta)$ and $m$ such that $(1 / m)<\delta-r$. If there exists $x_{0} \in \partial \bar{Q}_{r}$ such that $x_{0} \geq A_{m} x_{0}$, then for $t \in J_{1}$, we have

$$
\begin{aligned}
& \phi\left(x_{0}(t)\right) \\
& \geq \prod_{0<t_{i}<t}\left(1+\alpha_{i}\right) \int_{0}^{1} \frac{1}{\prod_{0<t_{i}<s}\left(1+\alpha_{i}\right)} \\
& \times G(t, s) \phi\left(f\left(s, x_{0}(s)+\frac{e}{m}\right)\right) d s \\
& \geq \prod_{0<t_{i}<t}\left(1+\alpha_{i}\right) \int_{J_{1}} \frac{1}{\prod_{0<t_{i}<s}\left(1+\alpha_{i}\right)} \\
& \times G(t, s)\left(\xi(s)-\varepsilon_{1}\right) \phi\left(x_{0}(s)+\frac{e}{m}\right) d s \\
& \geq \prod_{0<t_{i}<t}\left(1+\alpha_{i}\right) \int_{J_{1}} \frac{1}{\prod_{0<t_{i}<s}\left(1+\alpha_{i}\right)} \\
& \times G(t, s)\left(\xi(s)-\varepsilon_{1}\right) \phi\left(x_{0}(s)\right) d s \\
& \geq \frac{\sigma_{*}}{\sigma^{*}} \int_{J_{1}} G(t, s)\left(\xi(s)-\varepsilon_{1}\right) \phi\left(x_{0}\right) d s .
\end{aligned}
$$

Obviously,

$$
\inf _{t \in J_{1}}\left\|x_{0}(t)\right\| \geq \Omega(t)\left\|x_{0}\right\|_{P C}=\Omega(t) r>0 .
$$

The continuity of $x_{0}(t)$ implies that $\phi\left(x_{0}(t)\right)$ is continuous on $J_{1}$. Hence, this together with $(61)$ and $\left(H_{5}\right)$ implies that $\min _{t \in J_{1}} \phi\left(x_{0}(t)\right)>0$. Observing (59) and (60), then we can obtain a contradiction. Applying Lemma 4, we know that the fixed point index $i\left(A_{m}, Q_{r}, Q\right)=0$. Since $i\left(\theta, Q_{r}, Q\right)=1$, it follows from the homotopy invariance of fixed point index for strict set contraction that there exist $x_{m} \in \partial \bar{Q}_{r}$ and $0<$ $\lambda_{m}<1$ such that

$$
x_{m}=\lambda_{m} A_{m} x_{m} \text {, i.e. } A_{m} x_{m}=\lambda_{m}^{-1} x_{m}=\mu_{m} x_{m},
$$

where $\mu_{m}=\lambda_{m}^{-1}>1$. The rest of the proof is completely similar to that of Theorem 7. So it is omitted.
Example 10. Consider the following impulsive SBVP:

$$
\begin{gathered}
\mu x_{n}^{\prime \prime}+\frac{\pi}{\sqrt{t(1-t)}}\left(\frac{1}{3 n}\left(\sqrt{t} x_{n}+e^{2+x_{n+1}}\right)+\frac{\arctan t}{\sqrt{n}\|x\|}\right)=0 \\
t \in(0,1), \quad t \neq \frac{1}{5} \\
\left.\Delta x_{n}\right|_{t=1 / 5}=\frac{2}{5} x_{n} \\
2 x_{n}(0)-x_{n}^{\prime}(0)=\theta ; \\
x_{n}(1)=\theta, \quad(n=1,2,3, \ldots) .
\end{gathered}
$$

Conclusion. There exists a positive number $\delta$ such that for any given $r \in(0, \delta)$, impulsive $\operatorname{SBVP}(63)$ has a nontrivial, nonnegative eigenfunction $x_{r n}(n=1,2,3, \ldots)$ satisfying $\sup _{n} x_{r n}(t)<\infty$ for $0 \leq t \leq 1$ and $\max _{t \in[0,1]} \sup _{n} x_{r n}(t)=r$ corresponding to $\mu_{r} \geq 1$.

Proof. Let $E=l^{\infty}=\left\{x=\left(x_{1}, \ldots, x_{n}, \ldots\right): \sup _{n}\left|x_{n}\right|<\infty\right\}$ with norm $\|x\|=\sup _{n}\left|x_{n}\right|$ and $P=\left\{x=\left(x_{1}, \ldots, x_{n}, \ldots\right) \in\right.$ $\left.l^{\infty}: x_{n} \geq 0, n=1,2,3, \ldots\right\}$. Then, $P$ is a normal solid cone of $E$ with normal constant 1, and system (63) can be regarded as an impulsive SBVP in $E=l^{\infty}$ of the form (1), where $x(t)=$ $\left(x_{1}(t), x_{2}(t), x_{3}(t), \ldots\right), f(t, x)=\left(f_{1}, f_{2}, f_{3}, \ldots\right)$, and

$$
f_{n}(t, x)=\frac{\pi}{\sqrt{t(1-t)}}\left(\frac{1}{3 n}\left(\sqrt{t} x_{n}+e^{2+x_{n+1}}\right)+\frac{\arctan t}{\sqrt{n}\|x\|}\right) .
$$

It is easy to see that $f(t, x)$ is singular at $t=0,1$ and $x=\theta$.

Let $g(t)=\pi / \sqrt{t(1-t)}$ and $l(x)=\left(l_{1}(x), l_{2}(x), l_{3}(x), \ldots\right)$, where

$$
l_{n}(x)=\frac{1}{3 n}\left(x_{n}+e^{2+x_{n+1}}\right)+\frac{\pi}{4 \sqrt{n}\|x\|} .
$$

So we have

$$
\begin{gathered}
\|f(t, x)\| \leq g(t)\|l(x)\|, \quad t \in(0,1), \quad x \in P \backslash\{\theta\}, \\
l[r, R]=\sup _{x \in \bar{P}_{R} \backslash P_{r}}\|l(x)\|<R+e^{2+R}+\frac{\pi}{4 r}<+\infty,
\end{gathered}
$$

$$
\forall R>r>0 \text {. }
$$

By virtue of $\int_{0}^{1}(\pi / \sqrt{t(1-t)}) d t=\pi^{2}, G(s, s)=(1 / 3)(1+$ $2 s)(1-s)$, and

$$
\begin{gathered}
M_{0}=\max _{s \in J} G(s, s)=\frac{3}{8}, \\
\Omega(s)=\min \left\{\frac{1+2 s}{3}, 1-s\right\} \geq \frac{1}{3}(1+2 s)(1-s),
\end{gathered}
$$

we can obtain

$$
\begin{aligned}
\int_{0}^{1} G(s, s) g(s) l[\Omega(s) r, R] d s \\
\quad \leq \frac{3 \pi^{2}}{8}\left(R+e^{2+R}\right)+\frac{\pi^{3}}{4 r}<+\infty .
\end{aligned}
$$


So condition $\left(H_{1}\right)$ is satisfied. On the other hand, by the diagonal method of choosing subsequence, we can see that $L=0$ in condition $\left(\mathrm{H}_{2}\right)$; that is, condition $\left(\mathrm{H}_{2}\right)$ holds.

We now show that condition $\left(\mathrm{H}_{3}\right)$ is satisfied for

$$
\begin{gathered}
J_{0}=\left[\frac{1}{2}, \frac{9}{10}\right], \quad h(t)=\frac{1}{t}, \\
u_{0}=\left(2 \pi \arctan \frac{1}{2}, \frac{2}{\sqrt{2}} \pi \arctan \frac{1}{2}, \frac{2}{\sqrt{3}} \pi \arctan \frac{1}{2}, \ldots\right) .
\end{gathered}
$$

In fact,

$$
\begin{array}{r}
f_{n}(t, x) \geq \frac{\pi}{\sqrt{t(1-t)}} \frac{\arctan t}{\sqrt{n}\|x\|} \geq \frac{2}{\sqrt{n}} \pi \arctan \frac{1}{2} \cdot \frac{1}{\|x\|}, \\
\forall t \in\left[\frac{1}{2}, \frac{9}{10}\right],
\end{array}
$$

which means that condition $\left(H_{3}\right)$ is satisfied for

$$
\begin{gathered}
u_{0}=\left(2 \pi \arctan \frac{1}{2}, \frac{2}{\sqrt{2}} \pi \arctan \frac{1}{2}, \frac{2}{\sqrt{3}} \pi \arctan \frac{1}{2}, \ldots\right), \\
J_{0}=\left[\frac{1}{2}, \frac{9}{10}\right], \quad h(t)=\frac{1}{t}
\end{gathered}
$$

that is, condition $\left(\mathrm{H}_{3}\right)$ is satisfied.

Hence, our conclusion follows from Theorem 7.

\section{Conflict of Interests}

The authors declare that there is no conflict of interests regarding the publication of this paper.

\section{Acknowledgments}

The authors sincerely thank the reviewers for their useful comments and valuable suggestions on the original paper. This research is supported by NNSF of China (11171192) and Natural Science Foundation of Shandong Province (ZR2013AM005).

\section{References}

[1] Z. Wei, "Positive solution of singular Dirichlet boundary value problems for second order differential equation system," Journal of Mathematical Analysis and Applications, vol. 328, no. 2, pp. 1255-1267, 2007.

[2] Y. Liu and B. Yan, "Multiple solutions of singular boundary value problems for differential systems," Journal of Mathematical Analysis and Applications, vol. 287, no. 2, pp. 540-556, 2003.

[3] X. Xian, D. O'Regan, and C. Yanfang, "Structure of positive solution sets of semi-positone singular boundary value problems," Nonlinear Analysis: Theory, Methods \& Applications, vol. 72, no. 7-8, pp. 3535-3550, 2010.

[4] X. Zhang and M. Feng, "Positive solutions for a class of $2 n$ thorder singular boundary value problems," Nonlinear Analysis: Theory, Methods \& Applications, vol. 69, no. 4, pp. 1287-1298, 2008.
[5] R. P. Agarwal and D. O’Regan, "Nonlinear superlinear singular and nonsingular second order boundary value problems," Journal of Differential Equations, vol. 143, no. 1, pp. 60-95, 1998.

[6] D. Jiang, "Upper and lower solutions method and a superlinear singular boundary value problem," Computers \& Mathematics with Applications, vol. 44, no. 3-4, pp. 323-337, 2002.

[7] Z. Wei and C. Pang, "Positive solutions of nonresonant singular boundary value problem of second order differential equations," Nagoya Mathematical Journal, vol. 162, pp. 127-148, 2001.

[8] Y. Cui and Y. Zou, "Positive solutions of nonlinear singular boundary value problems in abstract spaces," Nonlinear Analysis: Theory, Methods \& Applications, vol. 69, no. 1, pp. 287-294, 2008.

[9] X. Xu and J. Ma, "A note on singular nonlinear boundary value problems," Journal of Mathematical Analysis and Applications, vol. 293, no. 1, pp. 108-124, 2004.

[10] S. Staněk, "Positive solutions of singular Dirichlet and periodic boundary value problems," Computers \& Mathematics with Applications, vol. 43, no. 6-7, pp. 681-692, 2002.

[11] X. Hao, L. Liu, Y. Wu, and N. Xu, "Multiple positive solutions for singular $n$ th-order nonlocal boundary value problems in Banach spaces," Computers \& Mathematics with Applications, vol. 61, no. 7, pp. 1880-1890, 2011.

[12] H. Su, L. Liu, and Y. Wu, "Positive solutions for Sturm-Liouville boundary value problems in a Banach space," Abstract and Applied Analysis, vol. 2012, Article ID 572172, 11 pages, 2012.

[13] X. Liu, W. Feng, and H. Liu, "Non-resonance and eigenvalues of nonlinear singular boundary value problems," Nonlinear Analysis: Theory, Methods \& Applications, vol. 50, no. 7, pp. 9951012, 2002.

[14] J. Henderson and W. Yin, "Positive solutions and nonlinear eigenvalue problems for functional differential equations," Applied Mathematics Letters, vol. 12, no. 2, pp. 63-68, 1999.

[15] X. Zhang and L. Liu, "Eigenvalue of fourth-order $m$-point boundary value problem with derivatives," Computers \& Mathematics with Applications, vol. 56, no. 1, pp. 172-185, 2008.

[16] D. Zhao and Y. Liu, "Eigenvalues of nonlinear singular boundary value problems," International Journal of Mathematics and Computation, vol. 22, no. 1, pp. 34-46, 2014.

[17] D. Guo, V. Lakshmikantham, and X. Liu, Nonlinear Integral Equations in Abstract Spaces, vol. 373, Kluwer Academic Publishers, Dordrecht, The Netherlands, 1996.

[18] B. Chanane, "Computing the eigenvalues of singular SturmLiouville problems using the regularized sampling method," Applied Mathematics and Computation, vol. 184, no. 2, pp. 972978, 2007.

[19] K. Q. Lan, "Multiple eigenvalues for singular Hammerstein integral equations with applications to boundary value problems," Journal of Computational and Applied Mathematics, vol. 189, no. 1-2, pp. 109-119, 2006.

[20] J. Ji and B. Yang, "Eigenvalue comparisons for boundary value problems for second order difference equations," Journal of Mathematical Analysis and Applications, vol. 320, no. 2, pp. 964972, 2006.

[21] J. Henderson and W. Yin, "Positive solutions and nonlinear eigenvalue problems for functional differential equations," Applied Mathematics Letters: An International Journal of Rapid Publication, vol. 12, no. 2, pp. 63-68, 1999.

[22] J. Suo and W. Wang, "Eigenvalues of a class of regular fourthorder Sturm-Liouville problems," Applied Mathematics and Computation, vol. 218, no. 19, pp. 9716-9729, 2012. 
[23] H. Coşkun and N. Bayram, "Asymptotics of eigenvalues for regular Sturm-Liouville problems with eigenvalue parameter in the boundary condition," Journal of Mathematical Analysis and Applications, vol. 306, no. 2, pp. 548-566, 2005.

[24] L. Aceto, P. Ghelardoni, and C. Magherini, "Boundary value methods as an extension of Numerov's method for SturmLiouville eigenvalue estimates," Applied Numerical Mathematics, vol. 59, no. 7, pp. 1644-1656, 2009.

[25] P. Ghelardoni, "Approximations of Sturm-Liouville eigenvalues using boundary value methods," Applied Numerical Mathematics, vol. 23, no. 3, pp. 311-325, 1997.

[26] S. Somali and V. Oger, "Improvement of eigenvalues of SturmLiouville problem with $t$-periodic boundary conditions," Journal of Computational and Applied Mathematics, vol. 180, no. 2, pp. 433-441, 2005.

[27] Q. Yao, "Positive solutions for eigenvalue problems of fourthorder elastic beam equations," Applied Mathematics Letters, vol. 17, no. 2, pp. 237-243, 2004.

[28] Z. Liu, J. Han, and L. Fang, "Integral boundary value problems for first order integro-differential equations with impulsive integral conditions," Computers \& Mathematics with Applications, vol. 61, no. 10, pp. 3035-3043, 2011.

[29] J. Tariboon, "Boundary value problems for first order functional differential equations with impulsive integral conditions," Journal of Computational and Applied Mathematics, vol. 234, no. 8, pp. 2411-2419, 2010.

[30] R. Liang and J. Shen, "Periodic boundary value problem for second-order impulsive functional differential equations," Applied Mathematics and Computation, vol. 193, no. 2, pp. 560571, 2007.

[31] X. Liu and D. Guo, "Method of upper and lower solutions for second-order impulsive integro-differential equations in a Banach space," Computers \& Mathematics with Applications, vol. 38, no. 3-4, pp. 213-223, 1999.

[32] K. Teng and C. Zhang, "Existence of solution to boundary value problem for impulsive differential equations," Nonlinear Analysis: Real World Applications, vol. 11, no. 5, pp. 4431-4441, 2010.

[33] L. Chen and J. Sun, "Boundary value problem of second order impulsive functional differential equations," Journal of Mathematical Analysis and Applications, vol. 323, no. 1, pp. 708$720,2006$.

[34] Y. Xu and H. Zhang, "Positive solutions of an infinite boundary value problem for nth-order nonlinear impulsive singular integro-differential equations in Banach spaces," Applied Mathematics and Computation, vol. 218, pp. 5806-5818, 2012.

[35] W. Li and G. Song, "Nonlinear boundary value problem for second order impulsive integro-differential equations of mixed type in Banach space," Computers \& Mathematics with Applications, vol. 56, no. 5, pp. 1372-1381, 2008.

[36] D. Guo, "Positive solutions of an infinite boundary value problem for $n$ th-order nonlinear impulsive singular integrodifferential equations in Banach spaces," Nonlinear Analysis: Theory, Methods \& Applications, vol. 70, no. 5, pp. 2078-2090, 2009. 


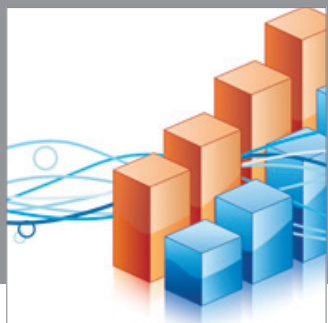

Advances in

Operations Research

mansans

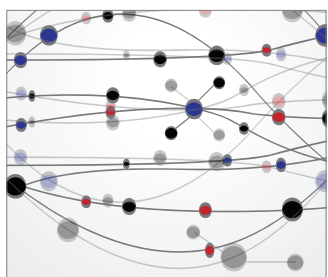

The Scientific World Journal
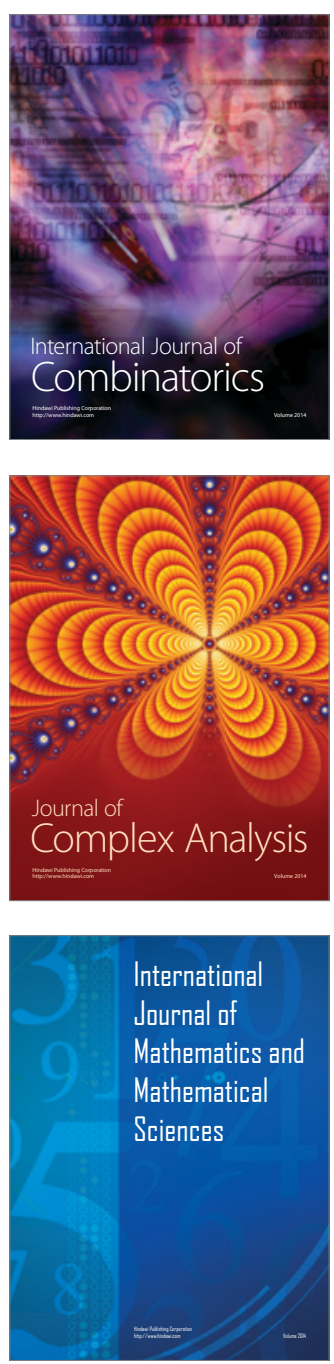
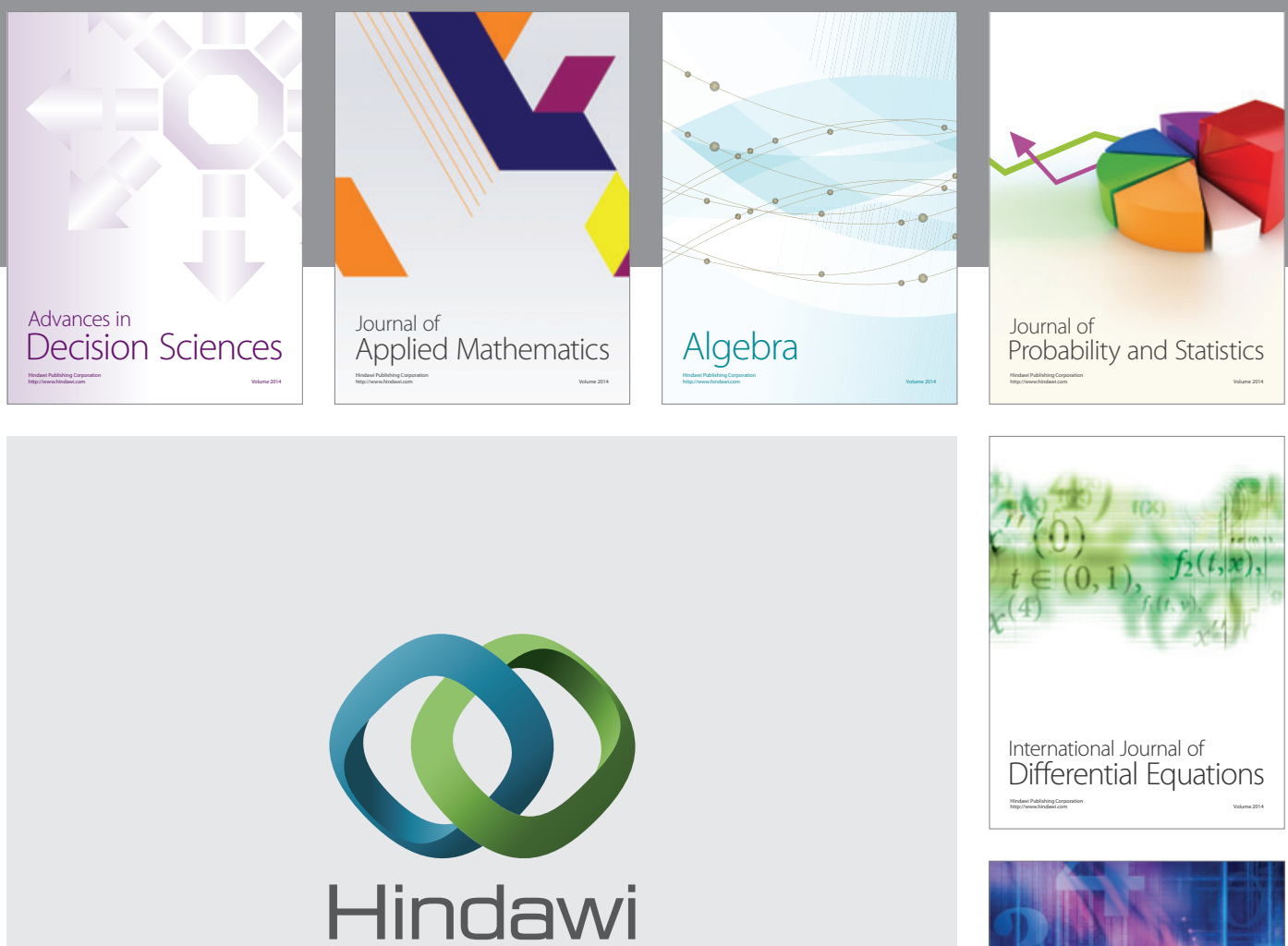

Submit your manuscripts at http://www.hindawi.com
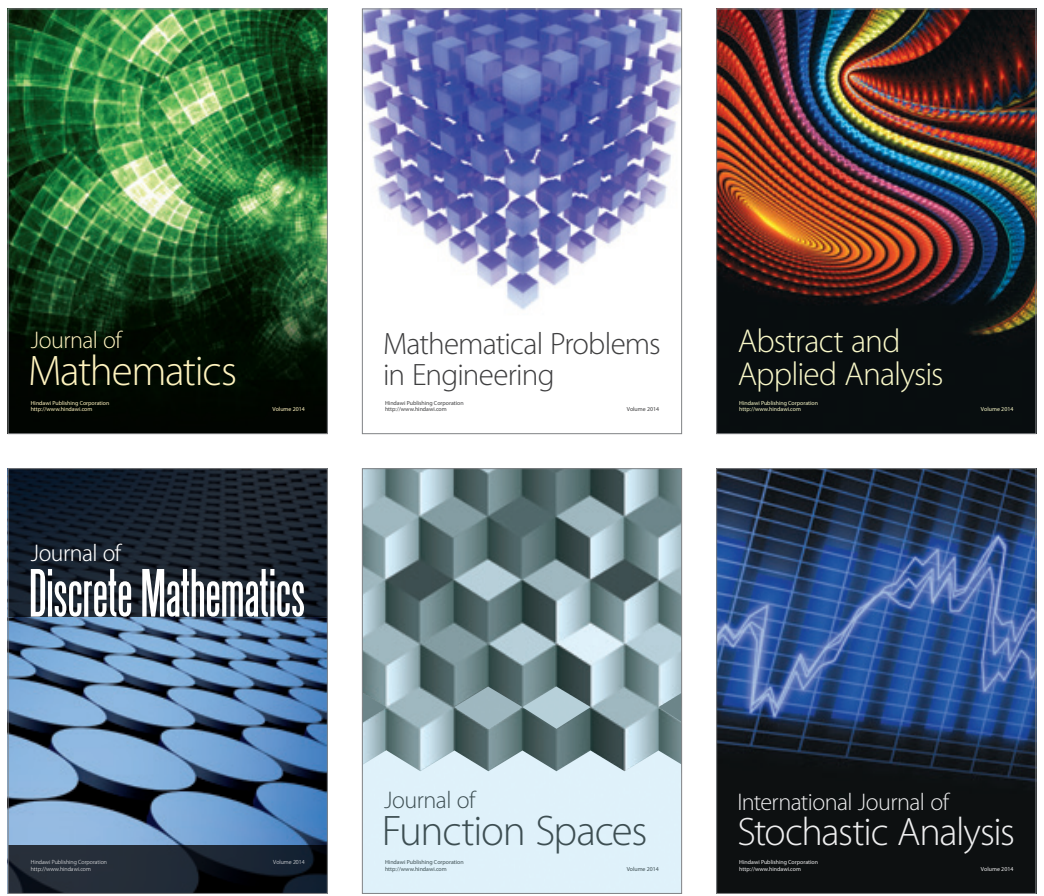

Journal of

Function Spaces

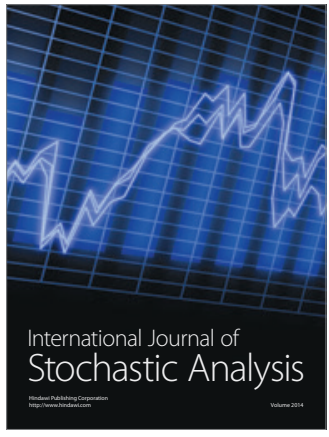

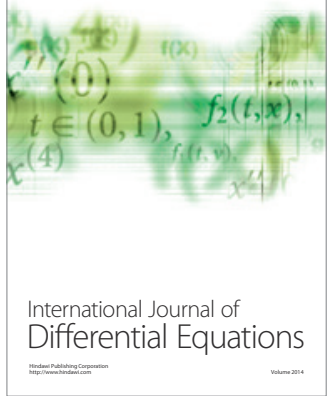
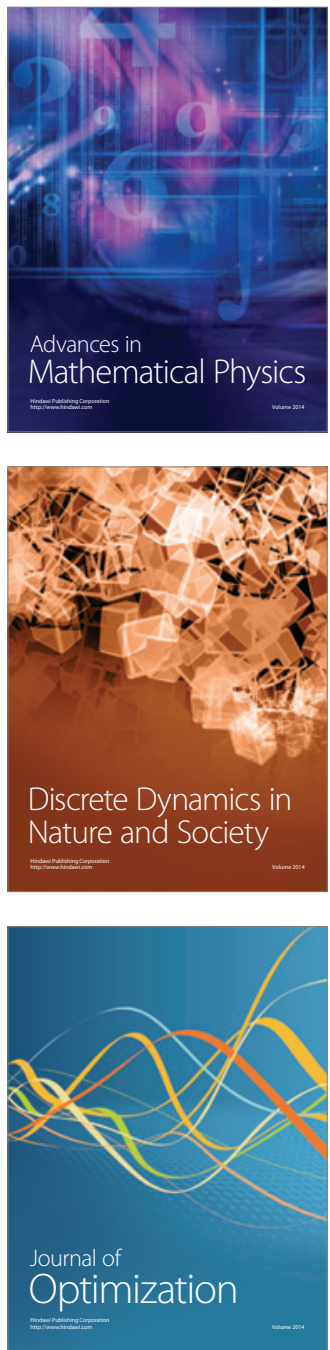